Conclusions Dyslipidemia is a significant comorbidity of LN that severely affects its renal and overall outcome. Its treatment represents a modifiable risk factor; adequate management can decrease its complications in LN patients and therefore improve their overall morbidity and mortality.

\section{PS8:170 LUPUS NEPHRITIS IN A MULTI- ETHNIC COHORT OF PATIENTS WITH SYSTEMIC LUPUS ERYTHEMATOSUS FROM BERKSHIRE, UK}

S Gindea, S Williams. Frimley Health Foundation Trust - Department of Rheumatology, Slough, UK

\subsection{6/lupus-2018-abstract.213}

Background Previous studies suggest that ethnicity influences the clinical phenotype in systemic lupus erythematosus (SLE), with lupus nephritis (LN) being more frequent in non-Caucasians. However, there are scarce data regarding prevalence of LN in South-Asian population (Indians, Pakistani, Sri Lanka, or Bangladeshi).

Objectives This study aimed to compare the prevalence of LN between lupus patients of different ethnicities, and to compare demographics and disease characteristics between LN patients. Methods This is a retrospective chart review study of 100 lupus patients followed from 2013 to 2017 at Wexham Park Hospital, a large district hospital in Southern England. The patients were categorised into four ethnic groups Caucasians, South-Asians, Blacks and Others (mixed race, Orientals, Arabs). LN prevalence, demographic and clinical data were compared using Fisher/Chi-Square tests for categorical variables and Wilcoxon test for continuous variables.

Results Of 100 patients in the study sample, 51\% were Caucasians, $31 \%$ were South-Asians, $11 \%$ were Blacks and $7 \%$ had other ethnicities. Mean age was 48 yo and $90 \%$ were females. Prevalence of LN was $26 \%$ in the full study sample and 24\%, 16\%, $64 \%$ and 57\%, respectively, among Caucasians, South-Asians, Blacks and Others. LN prevalence was significantly lower in Caucasians vs Blacks $(p=0.01)$, South-Asians vs Blacks $(<0.01)$ and South-Asians vs Other $(\mathrm{p}=0.02)$. Among patients with $\mathrm{LN}$, mean age was lower in South-Asians and Blacks, than Caucasians and Others (44 and 45 yo vs 52 and 51 yo). Blacks appear to include more males ( $43 \%$ vs $<25 \%$ in the other groups). Renal biopsy, available for 22/26 LN patients, suggested Class II predominance in Caucasians (44\% vs 33\% in the other groups). Proliferative LN (Class III and IV) was confirmed in 7 patients, without significant predominance in any ethnic group. Specific lupus autoantibodies (anti-dsDNA Ab and/or antiSm Ab) were found in 64\% Caucasians, $75 \%$ south-Asians, $86 \%$ Blacks and $75 \%$ others with LN.

Conclusion In our cohort, prevalence of $\mathrm{LN}$ in South-Asians was lower than in Blacks, but not statistically different comparing with Caucasians. However, South-Asians and Blacks with LN were younger than Caucasians. These results should be re-examined in larger similar multi-ethnic cohorts.

\section{PS8:171 ENDOTHELIAL DYSFUNCTION AND VASCULAR RISK FACTORS IN PATIENTS WITH SYSTEMIC LUPUS ERYTHEMATOSUS}

'A Martínez Zapico, ${ }^{2}$ Al Pérez Álvarez, 'L Caminal Montero, 'B Díaz López, ${ }^{2} \mathrm{~L}$ Benavente Fernández, ${ }^{1} \mathrm{R}$ Gómez de la Torre, ${ }^{1} \mathrm{D}$ Colunga Argüelles, ${ }^{3}$ J Rodríguez Carrio, ${ }^{3} \mathrm{P}$ López Suárez, ${ }^{3}$ A Suárez Díaz. 'Servicio de Medicina Interna. Hospital Universitario Central de Asturias, Oviedo- Asturias, Spain; ${ }^{2}$ Servicio de Neurología. Hospital Universitario Central de Asturias, Oviedo-Asturias, Spain; ${ }^{3}$ Área de Inmunología. Departamento de Biología Funcional. Universidad de Oviedo, Oviedo- Asturias, Spain

\subsection{6/lupus-2018-abstract.214}

Objectives Lupus systemic erythematosus is characterised by an increasing risk of premature cardiovascular disease (CVD). CVD is one of the most common causes of death in SLE. Subclinical atherosclerosis in comparison to general population is also more prevalent, especially the presence of plaques at the carotid level, as well as thickening of the carotid intima.

The aetiology of atherosclerotic disease is completely unknown. It involves: traditional risk factors (age, male gender, smoking, diabetes, hypertension, dyslipidemia, obesity) as well as risk factors related to the disease itself and the treatments used.

Methods A cross-sectional study was carried out from March to November 2015 in 119 patients. Patients were recruited from consultation at the Systemic Autoimmune Diseases Unit for a routine medical check. Clinical data on the disease (from diagnosis to the time of inclusion in the study) were obtained by reviewing the medical history.

The population was divided into two groups: patients with lupus and endothelial dysfunction and patients with lupus without endothelial dysfunction. The existence of endothelial dysfunction was explained by the presence of plaques at the carotid and/or intimate mean thickness $>0.8$ in a doppler ultrasonography.

Results There is no association with taking antimalarials, immunosuppressants, corticosteroids prior to high doses.

As for the classification criteria there is no relation with the presence of malar rash, Photosensitivity, Oral ulcers, Arthritis, Serositis, Nephropathy, Cytopenias and DNA.

No significant differences were detected in the determination of antibodies or complement levels.

No differences were found with SLEDAI. Since lupus is a disease that occurs in outbreaks, finding no differences may be due to the fact that at the time of inclusion patients had a low activity.

The presence of hypertension and dyslipidemia favours the existence of endothelial dysfunction. Hypertensive patients have a five-fold increased risk of developing endothelial dysfunction (5,593, 95\% CI: 2340 to 14,015$)$ as well as patients with dyslipidemia with a nearly 3 -fold increased risk (2,976 CI: 1191 to 7,591).

Conclusions Hypertension and dyslipidemia remain the classic risk factors associated with increased endothelial dysfunction. Strict control of them is imperative. 